\title{
The dark side of the QT interval.The Short QT Syndrome: pathophysiology, clinical presentation and management
}

\author{
I. Comelli*, G. Lippi**, G. Mossini***, G. Gonzi****, T. Meschi*, L. Borghi*, G. Cervellin*** \\ * Scuola di Specializzazione di Medicina d'Emergenza-Urgenza, Università degli Studi di Parma \\ ** U.O. Diagnostica Ematochimica, Azienda Ospedaliero-Universitaria di Parma \\ *** U.O. Pronto Soccorso e Medicina d'Urgenza, Azienda Ospedaliero-Universitaria di Parma \\ ***** U.O. Cardiologia, Azienda Ospedaliero-Universitaria di Parma
}

\section{Introduction}

A large number of studies has been carried out to investigate the pathophysiology and the clinical implications of QT interval prolongation in the ECG over recent years $(1,2,3,4,5,6)$. It was only in the last decade, however, that the scientists have focused on the specular aspects of the long QT syndrome (LQTS), and it is now well established that the abnormal shortening of the QT interval is associated with meaningful clinical consequences and adverse outcomes. The aim of the present article is to summarize knowledge and existing evidence about the Short QT Syndrome (SQTS). SQTS is a rare, albeit largely underdiagnosed, genetically determined disease, which is characterized by a high tendency to develop life-threatening arrhythmias. The two clinical landmarks of SQTS are the presence of a short QT interval (i.e., less than $320 \mathrm{~ms}$ ) in a structurally normal heart. The disease is now classified as a "channellopathy", and is principally caused by a defective functioning of both potassium and calcium ion channels. The underlying genetic anomalies cause an abnormal ripolarization and a reduced refractoriness of myocardiocites. Pharmacologic treatments are mainly tailored to slow the conduction and to prolong the refractory period of myocardiocites. The implantable cardioverter and defibrillator (ICD) is currently considered the therapeutic gold standard (7).

Keywords: short QT syndrome, channelopathies, sudden cardiac death.

\section{History}

In 1999 Gussak and colleagues firstly described some clinical cases characterized by shortening of the QT interval and electrical instability, thus suggesting the existence of a new clinical entity (8). In 2000, the same authors firstly described a family characterized by short QT interval. A female 51 years old and her two sons (17 and 21 years old, respectively) were found to have a "familial" shortening of T interval. The 17 years old girl displayed a QT interval of $280 \mathrm{~ms}$ during an episode of atrial fibrillation with a mean ventricular rate of 69 beats per minute, and a similar QT shortening was also identified in her mother and brother. The existence of a new familial syndrome, specular to the long QT syndrome (LQTS), was thereby conjectured (9). In 2003 the new clinical entity, defined as Short QT Syndrome (SQTS) has been categorically associated with the risk of sudden cardiac death (SCD), with description of two families with a high incidence of SDC, and also characterized as having a rest ECG with an abnormally shortened QT interval (10).

In 2004 and 2005 the first genetic mutations associated with three different subtypes of this new syndrome (i.e., SQTS1, SQTS2 and SQTS3) were described (Table 1) $(11,12,13)$. These mutations dramatically increase the rapid delayed rectifier potassium current $\mathrm{IKr}$ activity, thus shortening the action potential length and inducing a patchy pattern of refractoriness. In the same years a chinidine-based therapeutic approach has also been firstly introduced (14). In 2005 the first description of efficacy of Implantable Cardioverter and Defibrillator (ICD) in aborting SCD in a 16 years-old boy, with a familial history of SQTS, and with a basal QT interval of $252 \mathrm{~ms}$, has been provided (15). In 2007 two new genetic mutations (i.e., SQTS4 and SQTS5) associated with two different forms of disease have been described. Both SQTS4 and SQTS5 are characterized by ST elevation, with a Brugada-like pattern, in leads V1-V3, along with an abnormal shortening of the QT interval (16). Unlike Brugada syndrome and SQTS 1-3, which are respectively associated with anomalies of the sodium and potassium channels, these two new entities are linked to mutation of the CACNAIC and $C A C N B 2$ genes, that encode for the subunits $\alpha 1$ and $\beta 2 \mathrm{~b}$ of the calcium channels type $\mathrm{L}$. 
In 2011 Templin et al. identified a new mutation in the CACNA2D1 gene, causing a new subtype of the syndrome which was called SQTS6 and was characterized by a novel loss-of-function mutation coding for an L-type calcium channel subunit (17). In 2012 Hattori et al. identified another genetic mutation in the KCNJ2 gene, causing an abnormal function of the potassium channel (Table 1) (18).

Table 1. Association between genotype and phenotype of short QT syndrome (SQTS).

\begin{tabular}{|l|l|l|l|}
\hline Type & Gene involved & $\begin{array}{l}\text { Chromosome } \\
\text { locus }\end{array}$ & Reference \\
\hline SQTS1 & $\begin{array}{l}\text { KCNH2 (Potassium voltage-gated channel, subfamily H (eag-related), } \\
\text { member 2) }\end{array}$ & $1 \mathrm{q} 36.1$ \\
\hline SQTS2 & $\begin{array}{l}\text { KCNQ1 (Potassium voltage-gated channel, KQT-like subfamily, } \\
\text { member 1) }\end{array}$ & $11 \mathrm{p} 15.5-\mathrm{p} 15.4$ & 12 \\
\hline SQTS3 & $\begin{array}{l}\text { KCNJ2 (Potassium inwardly-rectifying channel, subfamily J, member } \\
\text { ) }\end{array}$ & $17 \mathrm{q} 24.3$ & 18 \\
\hline SQTS4 & $\begin{array}{l}\text { CACNA1C (Calcium channel, voltage-dependent, L type, alpha 1C } \\
\text { subunit) }\end{array}$ & $12 \mathrm{p} 13.3$ & 17 \\
\hline SQTS5 & $\begin{array}{l}\text { CACNB2 (Calcium channel, voltage-dependent, beta-2 subunit) } \\
\text { SQTS6 }\end{array}$ & $\begin{array}{l}\text { CACNA2D1 (Calcium channel, voltage-dependent, alpha-2/delta } \\
\text { subunit 1) }\end{array}$ & $7 \mathrm{q} 21.11$ \\
\hline
\end{tabular}

\section{Genetic background and physiopatological correlates}

The SQTS is a genetic disease with an autosomic dominant transmission, for which specific genetic mutations have been described, and are summarized in Table 1. Up to six different genotypes and phenotypes have been described so far. Nevertheless, as long as our knowledge about the pathophysiological bases of disease grows, it is conceivable that some others will be soon identified, as for Brugada syndrome (BS) which currently entails as many as eight different entities (from BS1 to BS8) (19). In general, all the genotypes associated with SQTS involve genetic polymorphisms and mutations in genes encoding structural glycoproteins of either potassium or calcium channels (Table 1). Basically, SQTS1, SQTS2 and SQTS3 are caused by gain-of-function mutations of cardiac potassium channels which are associated with hastened repolarization. The STQS1 is caused by genetic polymorphisms in the $K C N H 2$ gene which encodes potassium voltage-gated channel, subfamily $\mathrm{H}$ (eag-related), member 2 , and plays an essential role in the final repolarization of the ventricular action. Mutations in this gene may cause heterogeneous shortening of the action potentials and refractoriness (11). The STQS2 is associated with mutations of the $K C N Q 1$ gene, encoding the potassium voltage-gated channel, KQT-like subfamily, member 1. Mutations in this gene may cause a pronounced shift of the half-activation potential and an acceleration of the activation kinetics, finally leading to a gain of function of the protein (12).

The STQS3 is mainly associated with mutations in the KCNJ2 gene, which encodes for potassium inwardly-rectifying channel, subfamily J, member 2. Mutations in this gene can cause a gain of function in the strong inwardly rectifying channel protein, causing an accelerated repolarization process (13). Unlike SQTS1-3, the SQTS4-6 are mainly caused by loss of function mutations in genes encoding cardiac calcium channels. In cardiac myocytes, the calcium channels are composed of a pore-forming Cav1.2a1 subunit (encoded by the CACNA1C gene), and the two accessory subunits Cavb2b (encoded by the CACNB2b gene) and Cava2d-1 (encoded by the CACNA2D1 gene). In SQTS, the putative mutations induce a marked loss of function of the calcium channel, a decrease of intracellular calcium content, subsequent abbreviation of the action potential as well as ST-segment elevation (20).

Beyond families affected by SQTS, some sporadic cases with de novo mutations have been also described, which reflect the marked genotypic and phenotypic heterogeneity of this syndrome. Although the existence of a genotype-phenotype relationship has also been suggested, no therapeutic implications have been precisely defined as yet. Moreover, no clinically significant mutations or polymorphisms have been observed in several patients with SQTS $(21,22)$. As such, although a marked genetic heterogeneity characterizes SQTS as well as other inherited cardiac pathologies (e.g. Brugada and long QT syndromes, hypertrophic and dilated cardiomyopaties, see 19, 23), a "final common pathway" or 
"pattern" have consistently emerged, whereby genes encoding proteins with similar functions or involved in the same pathway are responsible for a particular disease or syndrome and channellopathies with increased risk of SCD may all share the hypothesis of the transmyocardial repolarization dispersion (TRD) $(24,25)$.

\section{Clinical presentation}

The clinical presentation and the course of SQTS are both rather heterogeneous. Although symptomatic tachyarrhytmias, syncopal episodes and SCD are the leading clinical markers of SQST, several cases may remain asymptomatic and thus unidentified. A high degree of phenotypic heterogeneity characterizes different families, but variable penetrance and expressivity have also been observed among members of the same family. Definitive relationships between stress, physical effort, sleep and SCD risk have not been found so far, nor convincing evidence has been provided supporting a role of autonomic nervous system in eliciting major arrhythmias.

The mean age at diagnosis is $\sim 30$ years, and the large majority of patients with a new diagnosis of SQTS has a family history of SCD, with a broad age distribution (i.e., between 3 months and 70 years). Recent data attest that $62 \%$ of SQTS patients have symptoms. The SCD is the first manifestation in $28 \%$ of cases. Palpitations (31\%), lightheadedness or syncope (24\%), and atrial fibrillation (17\%) are other frequent clinical features of SQTS. It is also conceivable that a substantial number of Sudden Infant Death Syndrome (SIDS) and prenatal deaths can be ascribed to SQTS (20, 21).

\section{Diagnostic criteria: the problem of measuring and interpreting the QT interval}

The diagnosis of this rare disease is challenging, but still critical to prevent SCD. The most critical problem is the lack of universal agreement for both diagnostic and prognostic criteria. An unequivocal definition of the lower threshold value for the QT interval is also lacking, and it cannot be excluded that some cases of the idiopathic ventricular fibrillation (VF) may be instead expression of a latent SQTS $(26,27)$ as reflected by the results of a retrospective study in subjects with aborted SCD due to VF, which showed that a short QT interval (i.e., $<300 \mathrm{msec}$.) may be present in up to $12 \%$ of such patients. A recent study of the European Registry of SQTS reported a $4.9 \%$ annual incidence of arrhythmic episodes in SQTS patients without antiarrhythmic therapy (28).

The QT interval is typically lengthened in a dynamic parameter, that physiologically decreases with increasing heart rate. It is also essential to distinguish the true SQTS from the frequent causes of physiological shortening of the QT interval, such as those occurring with sinus tachycardia, fever, acidosis, digoxin toxicity, hyperkalemia, hypocalcemia and hypercalcemia. The QT interval should be measured in II, aVF and left precordial leads, since those can clearly show U waves, even in presence of T wave abnormalities. A QT interval is usually considered "short" when its length is lower than 2 Standard Deviations (SD) of normal. These values have been specifically defined by Moss et al. in 1993 (29) by, as follows:

- Children: normal 370, range 310-420 msec. Short QT interval defined as $<310 \mathrm{msec}$.

- Men: normal 400, range 330-510 msec. Short QT interval defined as $<330 \mathrm{msec}$.

- Women: normal 390, range 330-480 msec. Short QT interval defined as $<330 \mathrm{msec}$.

It is important to mention here that the QT interval should always be corrected for the heart rate, and the classic Bazett's formula (i.e., correct QT interval [QTc] $=\mathrm{QT} / \sqrt{\mathrm{RR}}$ ) is now considered inappropriate for this purpose, because it's not accurate in the presence of bradycardia or tachycardia ${ }^{30}$. Luo and coworkers have suggested the additional lower limits of QT interval after comparing results of more than ten thousands ECGs with four different formulas (31):

- QTc Bazett: $363 \mathrm{msec}(<60 \mathrm{bpm}), 382 \mathrm{msec}(60-90 \mathrm{bpm})$;

- QTc Fridericia: $372 \mathrm{msec}(<60 \mathrm{bpm}), 370 \mathrm{msec}(60-90 \mathrm{bpm})$;

- QTc Framingham: $367 \mathrm{msec}(<60 \mathrm{bpm}), 374 \mathrm{msec}(60-90 \mathrm{bpm})$;

- QTc Hodges: $375 \mathrm{msec}$ (<60 bpm), $372 \mathrm{msec}(60-90 \mathrm{bpm})$.

In patients with SQTS patients, the ECG usually shows a significantly reduced QT interval, wherein values shorter than $300 \mathrm{msec}$. should immediately raise the clinical suspicion of disease. In precordial leads the $\mathrm{T}$ waves are often high, narrow and symmetric, although the T wave pattern can vary among different subtypes of the SQTS. It can be high, narrow and symmetric in SQTS1, symmetric but not narrowed in SQTS2, asymmetric in SQTS3. The ST segment is typically (extremely) short or even absent, with the $\mathrm{T}$ wave directly originating from the $\mathrm{S}$ wave. In some cases of the recently described SQTS4 and SQTS5 the shortened QT interval is associated with a Brugada-like elevation of the ST segment in leads V1-V3, both at rest and after administration of ajmaline (20,32). A high prevalence of early repolarization in SQTS subjects has also been occasionally described. This peculiar ECG pattern might be useful as an adjunctive tool for risk stratification of major events in SQTS patients (33). The lack of physiologic shortening of 
the QT interval is characteristic, and is typically observed in normal subjects when heart rate increases. It might hence be challenging, if not impossible, to diagnose SQTS with heart rate exceeding 100 beats per minute. In a significant percentage of SQTS patients, up to 53\%, atrial fibrillation (AF) might be the only clinically meaningful symptom at presentation. The exclusion of a shortened QT interval in young patients with "isolated" AF is thereby crucial (21). Due to the existence of a so-called "grey zone" of QTc intervals, where a substantial overlap between values of SQTS patients and those of the general (healthy) population occurs, it is crucial to rule out alternative diagnoses such as hyperkalemia, hypo- or hypercalcemia, sinus tachycardia, hypertermia, acidosis or digoxin therapy (22). As previously mentioned, the relative low number of SQTS cases is the leading reason for the uncertainty and poor agreement that still surrounds the definition of diagnostic criteria. Shulze et al. have suggested that the clinical suspicion of SQTS should be substantially raised in the presence of short QT interval associated with arrhythmias (especially AF or VF), and familial history of SCD, SQTS or both (34). Each case of SCD occurred at a young age underscores the possibility of a genetic-determined syndrome, and a thorough evaluation of all the family members is hence advisable (35). Unfortunately, only selected and highly specialized laboratories are currently equipped and organized for performing specific genetic testing, to the best of our knowledge, nor it is predictable that the routine assessment of those single nucleotide polymorphisms (SNPs) most commonly found in SQTS patients will be soon available outside highly-specialized or reference centers (36).

Table 2. Clinical scoring system for short QT syndrome (SQTS) developed by Gollob et al. (38).

\begin{tabular}{|c|c|c|c|}
\hline \multicolumn{2}{|c|}{ SQTS Diagnostic criteria } & \multirow{2}{*}{\begin{tabular}{|l} 
Points \\
1
\end{tabular}} & Probability \\
\hline \multirow{4}{*}{$\mathrm{QT}_{\mathrm{c}}, \mathrm{ms}$} & $<370$ & & \multirow{7}{*}{ High $>4$ points } \\
\hline & $<350$ & 2 & \\
\hline & $<330$ & 3 & \\
\hline & Jpoint-Tpeak interval $<120 \mathrm{~ms}$ & 1 & \\
\hline \multirow{4}{*}{$\begin{array}{l}\text { Clinical } \\
\text { History* }\end{array}$} & History of sudden cardiac arrest & 2 & \\
\hline & Documented polymorphic VT or VF & 2 & \\
\hline & Unexplained syncope & 1 & \\
\hline & Atrial fibrillation & 1 & \multirow{3}{*}{$2<$ Medium $<4$ points } \\
\hline \multirow{3}{*}{$\begin{array}{l}\text { Family } \\
\text { History* }\end{array}$} & First- or second-degree relative with high-probability SQTS & 2 & \\
\hline & $\begin{array}{l}\text { First- or second-degree relative with autopsy-negative sudden } \\
\text { cardiac death }\end{array}$ & 1 & \\
\hline & SIDS & 1 & \multirow{3}{*}{ Low $<2$ points } \\
\hline \multirow[b]{2}{*}{ Genotype* } & Genotype positive & 2 & \\
\hline & Mutation of undetermined significance in a culprit gene & 1 & \\
\hline \multicolumn{4}{|c|}{$\begin{array}{l}\text { *A minimum of } 1 \text { point must be obtained in the electrocardiographic section in order to obtain additional points. } \\
\text { Electrocardiogram: must be recorded in the absence of modifiers known to shorten the QT. Jpoint-Tpeak interval } \\
\text { must be measured in the precordial lead with the greatest amplitude T-wave. } \\
\text { Clinical history: events must occur in the absence of an identifiable etiology, including structural heart disease. Points } \\
\text { can only be received for } 1 \text { of cardiac arrest, documented polymorphic VT, or unexplained syncope. } \\
\text { Family history: points can only be received once in this section. }\end{array}$} \\
\hline
\end{tabular}

The Guidelines of the Joint Steering Committees of UCARE and IVF-US currently advocates a basic evaluation of suspected individuals including baseline ECG, stress testing, echocardiography, Holter monitoring and nuclear magnetic resonance (NMR) of the heart for eventually excluding the presence of a structural cardiomyopathy. A thorough 
personal and familial history still represent the cornerstone of the clinical evaluation, wherein a short QT interval associated with arrhythmic symptoms (isolated AF or aborted VF), and familial history of SCD, SQTS or both is extremely suggestive for SQTS $(20,37)$.

In analogy with the LQTS, a clinical scoring system based on the first 61 reported cases has been developed by Gollob et al. (Table 2) (38). This scoring system, however, has not yet been widely accepted. It is also noteworthy that Bjerregaard recently emphasized that SCD cases have not been described so far for QTc intervals comprised between 340 and $350 \mathrm{~ms}$, so that major caution should be used before recommending its routine introduction in clinical practice (39). The prognosis of asymptomatic cases of SQTS is currently uncertain (40).

\section{Clinical management}

The pharmacologic approach to SQTS is mainly targeted at prolonging the repolarization, by blockage of potassium efflux. Some QT-prolonging drugs, which are implicitly harmful in LQTS, can however be life-saving in SQTS. Gaita et al. assessed four antiarrhytmic drugs (i.e., flecainide, sotalol, ibutilide and chinidine) in six SQTS patients, and found that only Chinidine was really effective to prolong the QT interval up to normal values, thus reducing the refractory period as well as the inducibility of life-threatening arrhythmias (41). IC and III class drugs failed to show comparable efficacy. The patients treated with chinidine for one year remained asymptomatic, without further diagnosed arrhytmias. Due to the current lack of solid criteria for risk stratification, oral chinidine represents an acceptable alternative to implantable cardioverter-defibrillators (ICD) in asymptomatic patients. A recent study, based on the European Registry of the SQTS, has confirmed the lack of adverse events at follow-up in 12 SQTS patients taking chinidine for a mean follow-up period of 64 months $(41,42,43)$. The gold standard for treatment of symptomatic SQTS is represented by ICD for patients with history of aborted SCD due to VF or VT (class I; level of evidence B), and for patients with a familial history of SCD and induction of VF (class II; level of evidence B) $(32,44,45)$. Implantation of an ICD is recommended in all patients with SQTS for primary prevention of SCD, unless contraindicated or declined by the patient (46, 47, 48). Some inappropriate ICD discharge due to T-wave oversensing has been linked with suicide in young SQTS patients, hence the risk stratification of patients must be very accurate before this clinical decision (49).

\section{Conclusions}

SCD is typically defined as natural death due to cardiac causes, anticipated by a sudden loss of consciousness, within one hour from the beginning of symptoms. The incidence is comprised between 0.36 and 1.28/1000 citizens/year (e.g., $\sim 300,000$ cases each year in the US), which makes it one among the leading causes of death in the western world. The vast majority of cases is attributable to structural cardiac diseases, coronary artery disease being the leading one. However, a non negligible 5-10\% of all cases occur in young individuals, without a diagnosed structural heart disease. Among the causes of SCD in young people, the SQTS is relatively recent, being described only ten years ago. Like BS and LQTS, SQTS belongs to a family of genetic disease known as "channellopathies". Although it can be considered globally rare, SQTS afflicts more often young and apparently healthy people and is a life-threatening condition, which should raise the level of alertness of clinicians. Emergency physicians should be particularly aware that the incidental finding of a short QT interval in young subjects should not be underestimated, especially when associated with arrhythmic symptoms, syncope or documented AF, both paroxystic and persistent. Any suspected case should be specifically addressed to specialized centers, where it should be managed by a multidisciplinary team, in which cardiologists, psychologists and genetists would join their specific knowledge to prevent or limit the risk of SCD in young persons. It is also noteworthy, however, that the lack of a robust genotype-phenotype correlation in SQTS still implies a primarily clinical management, since a therapeutic approach mainly based on genetic or biochemical findings would be ineffective or even inappropriate in several patients.

\section{Abbreviations}

CPVT: catecholaminergic polymorphic ventricular tachycardia

DS: standard deviations

ECG: electrocardiogram

FA: atrial fibrillation

VF: ventricular fibrillation

ICD: cardioverter implantable defibrillator

LQTS: long QT syndrome 
QTc: correct QT interval

QTp: predict QT interval

SCD: sudden cardiac death

SIDS: sudden infant death syndrome

SQTS: short QT syndrome

TDR: trans myocardial dispersion of repolarization

VT: ventricular tachycardia

\section{References}

1. Ward OC. A new familial cardiac syndrome in children. Irish Med J 1964; 54: 103-6.

2. Schwartz PJ, Stramba-Badiale M, Crotti L et al. Prevalence of the congenital long-QT syndrome. Circulation 2009; 120: 1761-7.

3. Priori SG, Schwartz PJ, Napolitano C. Risk Stratification in the Long-QT Syndrome. N Engl J Med 2003; 348: 1866-74.

4. Sauer AJ, Moss AJ, McNitt S et al. Long QT syndrome in adults. J Am Coll Cardiol 2007; 49: 329-37.

5. Roden DM. Drug-Induced Prolongation of the QT Interval. N Engl J Med 2004; 350: 1013-22.

6. Comelli I, Cervellin G, Meschi T et al. When it lengthens, can shorten your life. On the multifaceted aspects of long QT. Emerg Care J 2011; 4: 19-28.

7. Cross B, Homoud M, Link M et al. The short QT syndrome. J Interv Card Electrophysiol 2011; 31: 25-31.

8. Gussak I, Liebl N, Nouri S et al. Deceleration-Dependent Shortening of the QT interval: A New Electrocardiographic Phenomenon? Clin Cardiol 1999; 22: 1124-26.

9. Gussak I, Brugada P, Brugada J et al. Idiopathic Short QT Interval: A New Clinical Syndrome? Cardiology 2000; 94: 99-102.

10. Schrimpf R, Wolpert C, Bianchi F et al. Congenital Short QT Syndrome and Implantable Cardioverter Defibrillator Treatment: Inherent Risk for Inappropriate Shock Delivery. J Cardiovasc Electrophysiol 2003; 14: 1273-77.

11. Brugada R, Hong K, Dumaine R et al. Sudden Death Associated With Short-QT Syndrome Linked to Mutations in HERG. Circulation 2004, 109: 30-35.

12. Bellocq C, van Ginneken ACG, Bezzina CR et al. Mutation in the KCNQ1 Gene Leading to the Short QT-Interval Syndrome. Circulation 2004; 109: 2394-97.

13. Priori SG, Pandit SV, Rivolta I et al. A novel form of short QT syndrome (SQT3) is caused by a mutation in the KCNJ2 gene. Circ Res 2005; 96: 800-7.

14. Wolpert C, Schimpf R, Giustetto C et al. Further insights into the effect of quinidine in short QT syndrome caused by a mutation in HERG. J Cardiovasc Electrophysiol 2005; 16: 54-8.

15. Schimpf R. Short QT syndrome: successful prevention of sudden cardiac death in an adolescent by implantable cardioverter-defibrillator treatment for primary prophylaxis. Heart Rhythm 2005; 2: 416-7.

16. Antzelevich C, Pollevick G, Cordeiro J et al. Loss-of-function mutations in the cardiac calcium channel underlie a new clinical entity characterized by ST-segment elevation, short QT intervals, and sudden cardiac death. Circulation 2007; 115: 442-49.

17. Templin C, Ghadri JR, Rougier JS et al. Identification of a novel loss-of-function calcium channel gene mutation in short QT syndrome (SQTS6). European Heart Journal 2011; 32: 1077-88.

18. Hattori T, Makiyama T, Akao M et al. A novel gain-of-function KCNJ2 mutation associated with short-QT syndrome impairs inward rectification of Kir2.1 currents. Cardiovasc Res 2012; 4: 666-73.

19. Lippi G, Montagnana M, Meschi T et al. Genetic and clinical aspects of Brugada syndrome: an update. Adv Clin Chem 2012; 56: 197-208.

20. Patel C, Yan GX, Antzelevitch C. Short QT Syndrome: From Bench to Bedside. Circ Arrhythm Electrophysiol 2010; 3: 401-8.

21. Borggrefe M, Wolpert C, Antzelevitch C et al. Short QT sindrome. Genotype-phenotype correlations. Journal of Electrocardiology 2005; 38(suppl.1): 75-80.

22. Brugada R, Hong K, Cordeiro JM et al. Short QT syndrome. CMAJ 2005; 173 (11): 1349-54.

23. Lippi G, Targher G, Franchini M, Plebani M. Genetic and biochemical heterogeneity of cardiac troponins: clinical and laboratory implications. Clin Chem Lab Med 2009; 47: 1183-94.

24. Bowles NE, Bowles KR, Towbin JA. The 'final common pathway hypothesis' and inherited cardiovascular disease. Herz, 2000, 25 (3):168-75. 
25. Antzelevich C. Heterogeneity and cardiac arrhythmias: an overview. Heart Rhythm 2007; 4, 964-972.

26. Viskin S, Zeltser D, Shalom MI et al. Is idiopathic ventricular fibrillation a short QT syndrome? Comparison of QT intervals of patients with idiopathic ventricular fibrillation and healthy controls. Heart Rhythm 2004; 1: 587-91.

27. Crotti L, Taravelli E, Girardengo G et al. Congenital short QT syndrome. Indian Pacing Electrophysiol J. 2010; 10: $86-95$.

28. Giustetto C, Schimpf R, Mazzanti A et al. Long-term follow-up of patients with short QT syndrome. J Am Coll Cardiol 2011; 58: 587-95.

29. Moss AJ. Measurement of the QT interval and the risk associated with QTc interval prolongation: A review. Am $J$ Cardiol 1993; 72: 23-25.

30. Bazett H. An analysis of the time relationship of electrocardiograms. Heart 1920; 7: 353-70.

31. Luo S, Tompkins WJ. Parameter evaluation of the inverse power-law spectrum of heart rate. A quantitative approach for ECG arrhythmia analysis. J Electrocardiol 1994; 27: 46-52.

32. Lunati M, Santini M, Bongiorni MG et al. Linee guida AIAC all'impianto di pacemaker, dispositivi per la resincronizzazione cardiaca, defibrillatori automatici e loop recorder update 2011. GIAC 2011; 14: 1-55.

33. Watanabe H, Makiyama T, Koyama T et al. High prevalence of early repolarization in short QT syndrome. Heart Rhythm 2010; 5: 647-52.

34. Schulze-Bahr E, Breeithardt G. Short QT Interval and Short QT Syndromes. Journal of Cardiovascular Electrophysiology 2005; 16: 397-98.

35. Brugada R. Sudden death: managing the family, the role of genetics. Heart 2011; 97: 676-81.

36. Redpath CJ, Green MS, Birnie DH et al. Rapid genetic testing facilitating the diagnosis of short QT syndrome. Can J Cardiol 2009; 25: 133-35.

37. Joint Steering Committees of UCARE and IVF-US. Survivors of out-of hospital cardiac arrest with apparently normal heart. Need for definition and standardized clinical evaluation. Consensus Statement of the Joint Steering Committees of the Unexplained Cardiac Arrest Registry of Europe and of the Idiopathic Ventricular Fibrillation Registry of the United States. Circulation 1997; 95: 265-72.

38. Gollob MH, Redpath CJ, Roberts JD. The Short QT Syndrome: Proposed Diagnostic Criteria. J Am Coll Cardiol 2011; 11: 802-12.

39. Bjerregaard P. Proposed Diagnostic Criteria for Short QT Syndrome Are Badly Founded. J Am Coll Cardiol 2011; 58: 549-50.

40. Kobza R. Identifying risk of cardiac events in short QT syndrome. Heart Rhythm 2012; 9: 66-74.

41. Gaita F, Giustetto C, Bianchi F et al. Short QT syndrome: pharmacological treatment. J Am Coll Cardiol 2004; 43: 1494-99.

42. Giustetto C, Schimpf R, Mazzanti A et al. Long-term follow-up of patients with short QT syndrome. J Am Coll Cardiol 2011; 58: 587-95.

43. Patelb C, Antzelevitch C. Pharmacological approach to the treatment of long and short QT syndrome. Pharmacol Ther 2008; 118: 138-51.

44. Giustetto C, Wolpert C, Borggrefe M et al. Short QT syndrome: clinical findings and diagnostic-therapeutic implications. Eur Heart J 2006; 27: 2382-84.

45. Stephenson EA, Berul CI. Electrophysiological Interventions for Inherited Arrhythmia Syndromes. Circulation 2007; 116: 1062-80.

46. Bjerregaard P, Gussak I. Short QT syndrome. Ann Non Invasive Electrocardiol 2005; 10: 436-40.

47. Schimpf R, Bauersfeld U, Gaita F et al. Inappropriate Inplantable Cardioverter Defibrillator Therapy in the Short QT Syndrome: Old Problem in a New Disease. J Cardiovasc Electrophysiol 2003; 14: 1278-79.

48. Zipes DP, Camm AJ, Borggrefe M et al. ACC/AHA/ESC 2006. Guidelines for Management of Patients With Ventricular Arrhythmias and the Prevention of Sudden Cardiac Death. Circulation 2006; 114: 385-484.

49. Sun Y, Ping Zhang P, Li X et al. Inappropriate ICD Discharge Due to T-Wave Oversensing in a Patient with Short QT Syndrome. Pacing Clin Electophys 2009; 33: 113-6. 